\title{
Polarization and extremism emerge from rational choice
}

\author{
Peter D. Kvam \\ University of Florida
}

\author{
Matthew Baldwin \\ University of Florida
}

\begin{abstract}
Polarization is often thought to be the product of biased information search, motivated reasoning, or other psychological biases. However, polarization and extremism can still occur in the absence of any bias or irrational thinking. In this paper, we show that polarization occurs among groups of decision makers who are implementing rational choice strategies that maximize decision efficiency. This occurs because extreme information enables decision makers to make up their minds and stop considering new information, whereas moderate information is unlikely to trigger a decision. Furthermore, groups of decision makers will generate extremists - individuals who hold strong views despite being uninformed and impulsive. In re-analyses of seven previous empirical studies on both perceptual and preferential choice, we show that both polarization and extremism manifest across a wide variety of choice paradigms. We conclude by offering theoretically-motivated interventions that could reduce polarization and extremism by altering the incentives people have when gathering information.
\end{abstract}

Attitudes toward crucial economic, environmental, and social policies are becoming alarmingly and increasingly polarized (Baldassarri \& Gelman, 2008; Wilson et al., 2020). Data from large-scale social surveys, such as the American National Election Study, show that self-identified Republicans and Democrats are relatively split on a variety of issues, and these splits are apparent for activists, partisans and ideologues, as well as voters (Baldassarri \& Gelman, 2008). The political divide is obvious, but the reasons for the divide are up for some debate. As we review the following sections, previous work on political polarization has focused on the motivational and social forces that can deepen divisions between groups. Despite these potential contributions to polarization, neither motivated reasoning nor social interactions are necessary for polarization to occur. On the contrary, we show that perfectly rational individual decision makers - whose goals are to make accurate and efficient choices and who need not be subject to social influence - can still become polarized.

\section{Known sources of polarization}

People tend to seek out information that will confirm their existing beliefs (Festinger, 1957; Nickerson, 1998), illustrated in a tendency to select and engage with news articles that align with their existing beliefs from both unbiased and slanted news sources (Westerwick et al., 2017). Managing the dissonance that arises from counter-attitudinal information by seeking out the pleasure of confirmatory information leads to greater polarization, as decision makers with opposing attitudes begin to deal in the extreme ends of the information distribution. Common ground is destroyed, and worse, extreme social distance is created as political information is selected not to inform but rather to reinforce negative atti- tudes toward one's opponents (Iyengar et al., 2012).

In concert with these confirmation biases, people also strive to disconfirm or discount information that is not in line with their existing beliefs. In the off-chance that one does see counter-attitudinal information, decision makers appear unable to ignore their prior beliefs when processing that information (Taber et al., 2009). In a similar vein, experimental findings have shown that groups of US Twitter users on opposite sides of the political spectrum became more polarized when exposed to opposing political views (Bail et al., 2018). In dealing with the negative affect, or dissonance, associated with exposure to disconfirming political information, decision makers appear to either attempt to disconfirm that information or seek other information that aligns with their existing beliefs.

Polarization is also reinforced when people conform to in-group norms and values, both because doing so provides individuals with a clear and positive identity (H. E. Tajfel, 1978; H. Tajfel \& Turner, 1986) and for the interpersonal benefits that practicing in-group norms confers (Hogg \& Turner, 1985; Suhay, 2015). Those who "stick with the group" and practice in-group norms and values tend to be treated with respect which leads to feelings of pride (Lewis, 2008), whereas those to "betray the group" tend to be shamed, leading to embarrassment (Milgram et al., 1992). When group norms are made salient-such as a political party's stance on a social issue-group members tend to bring to mind extreme prototypical information about the issue, as doing so helps to distinguish the in-group from the out-group (Messick \& Mackie, 1989).

Of course, not all polarization occurs from purely psychological mechanisms. A growing body of research has demonstrated the impact that social media-and media in general- 
is having on immense groups of people world-wide. The goal of media has shifted from slow, objective journalism to fast sensationalized news that competes for the attention of users (Foer, 2017; Klein, 2020). Selective reporting that serves the purpose of capturing attention focuses on extreme events, often those that paint one side of the ideological spectrum in a negative light. Thus, the media landscape is ripe for satisfying the biases mentioned previously-finding and sharing confirmatory information is easy, quick, and spreads rapidly which leads to false perceptions of extremely polarized groups (Ahler \& Sood, 2018). Moreover, this information tends to produce moral outrage as it spreads on social media, which is used as a platform to announce one's allegiance to certain groups, ideas, and movements as well as derogate those who are different (Brady et al., 2017; Crockett, 2017). The consequence is that social media algorithms learn to make this information go viral, often creating echo chambers of misleading, or outright false, information (Benkler et al., 2018), which of course can be polarizing.

\section{The rational decision maker}

This work paints a picture of political and affective polarization that arises from biases in information processing or belief updating (DeKay, 2015; DeKay et al., 2009), motivation (Festinger, 1957; Nickerson, 1998), group processes (Hogg \& Turner, 1985; Suhay, 2015), and even media algorithms and trends (Benkler et al., 2018). Each of these explanations for polarization implicitly or explicitly assumes that polarization occurs because of fundamental biases in the in the information decision makers encounter or irrationality in the way they make decisions. As a result, we could be led to believe that these divides would not come about if decision makers gathered representative information and considered it at face value. However, it is not necessary for decision makers to act irrationally or gather biased information for polarization to occur. Groups can become polarized and individuals become extremists even when everyone implements optimal or rational choice strategies that maximize decision quality and efficiency.

When a decision maker is presented with a choice between two (or more) options, they must make a compromise between investing time on that decision - in order to achieve better outcomes by considering more information - and making a selection so that they can move onto their next choice. The optimal way to make these decisions is to set a threshold that determines the desired level of accuracy or satisfaction that the decision maker desires, and then gather information until that threshold level of evidence is reached (Bogacz et al., 2006, 2010; Edwards, 1965). Nearly all models of dynamic decision making are based on this principle: sequential sampling and random walk / diffusion processes are commonly used to describe and explain human choice behavior (Ratcliff et al., 2016; Busemeyer et al., 2019) and the associ- ated neural activity (Gold \& Shadlen, 2007; Heitz \& Schall, 2012) across a wide variety of perceptual and preferential domains. These models are based on the sequential probability ratio test (Wald \& Wolfowitz, 1949; Edwards, 1965), where a decision maker tracks the balance of support between two options and uses this balance to determine when to make a choice. As they get information favoring option A, they shift up toward the "Choose A" response boundary, as shown in Figure 1. As they get information favoring option B, they shift down toward the "Choose B" response boundary. Once they cross one boundary or the other, option A or B is chosen.

The key element of this decision process related to polarization is that the goal of a decision maker is not to gather a representative sample of information, but to gather enough information to conclude $A \succ B$ or $B \succ A$ (where $\succ$ denotes a preference order). The difference in these two goals was examined in work by Coenen \& Gureckis (2016), where participants were presented with a deck of cards containing blue and red cards. Participants' task was either to make a choice about whether there were more red or blue cards in the deck (choice condition) or estimate the proportion of red / blue cards in the deck (estimation condition). Critically, Coenen \& Gureckis found that the distribution of samples gathered by participants in the choice condition was not representative of the actual proportion of red cards in the deck. Instead, participants appeared to have over-sampled red cards if they had chosen red, or over-sampled blue cards if they had chosen blue.

On one hand, this is not so surprising, because we should expect participants to have choice-consistent samples: those who sample more red cards should choose red, and those that sample more blue cards should choose blue. But underneath this obvious pattern was one more surprising; namely, that the cards that participants had viewed were not representative of the proportion of cards in the decks. Somewhere between the perfectly representative samples that participants were receiving and the samples that participants had at the end, the sample information had become more extreme. The critical observation from this study is that the goals of the decision makers influence the final sample of information that they collect. Even decision makers approximating the optimal strategy appeared to end their choices with unrepresentative samples of information about the underlying distribution of red and blue cards.

One relatively intuitive reason this occurs is because moderate information is not conducive to achieving an agent's decision goals (to stop and support A over B, or B over A), while extreme information makes it quite easy to select one option over the other. As a result, a decision maker is more likely to stop gathering new information after sampling a particularly extreme piece of information than they are to stop after sampling a moderate piece of information. This idea is illustrated in the top panel of Figure 1: moderate information 


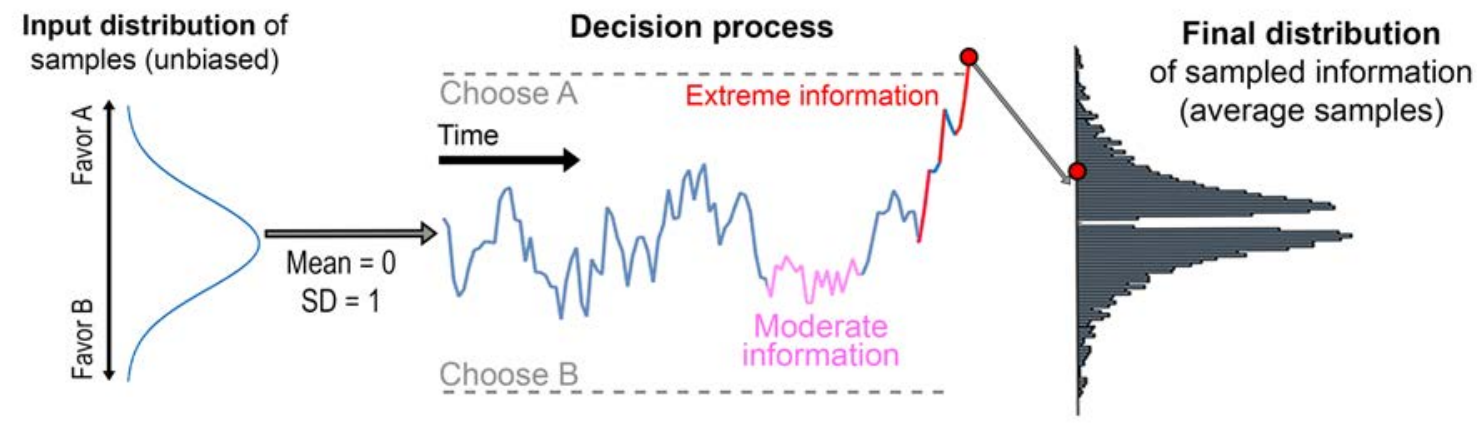

\section{Figure 1}

Illustration of how an unbiased distribution of information (left) becomes a polarized distribution (right) when fed through the decision process (middle).

(pink) cannot tip the balance of evidence sufficiently toward one option or another to trigger a choice, while extreme information (red) is likely to do so. As a result, people who have made a decision using a relative balance-of-evidence stopping rule will have a greater number of extreme information samples than the underlying distribution.

The consequence of this property of information is that individuals can become polarized when making optimal choices, when there is no bias in the information they collect or how they interpret it, and when they have no initial motivation to favor one option over another. A formal proof, deriving the statistical distribution of information that decision makers will have collected at the time of choice, is provided in the supplementary information. This shows the generality of our conclusions - for any input information distribution, it allows us to derive the final distribution of information in a population of decision makers. Furthermore, we show that optimal decision processes create individual "extremists" who gather very little information and yet hold much more extreme views than other decision makers. These decision makers tend to value time over new information relative to other decision makers, reflected in lower thresholds that generate fast but not necessarily accurate responses (Heitz \& Schall, 2012; Wickelgren, 1977).

In the following sections, we describe the results of reanalyses of seven empirical studies as they relate to polarization and extremism resulting from rational choice strategies, and discuss the implications alongside those of the simulations and proof presented in the supplement. We show that the incentives for making decisions create groups of decision makers whose beliefs and opinions are more polarized than the true underlying pools of information from which they are sampling. Furthermore, we show that these groups will naturally contain extremists who have the least information out of all decision makers but the most extreme beliefs and opinions. These extremists are able to dominate subsequent discourse among the population of decision makers, both because they make their decisions fastest (and thus will be the first to disseminate the information they have gathered) and carry disproportionate influence over the cultural transmission of opinions and beliefs (Navarro et al., 2018).

\section{Results}

To examine whether polarization and extremism appeared in empirical work, we analyzed the results of seven different decision making tasks. These tasks varied in the type of stimuli and decision domain (preferential, inferential), but they had three important characteristics in common that made it possible to examine polarization and extremism: (1) the main goal of participants in the experiments was to make a series of decisions between binary choice options; (2) the true information provided by the stimulus was known, and tracked by the experimenters; and (3) each experiment recorded the final (average) set of information that participants had gathered on each trial. The seven experiments we analyzed were:

- Study 1 (Dots): Decisions between two dot colors. Participants had to determine whether sequentially presented dots, each of which was pink or green, was drawn from a pool of dots that was $2 / 3$ pink ( $1 / 3$ green) or $2 / 3$ green (1/3 pink).

- Study 2 (Currency): Decisions between two collections of currencies. Participants were presented with two sets of money, where each set was split into three different currencies (e.g., \$, $¥, Q$ ), . Their task was to decide which set of money they would prefer to draw from, given that they would randomly receive one of the three currencies in the set.

- Study 3 (Food): Participants decided which of two food options they would prefer to receive. They had previously rated the quality of both food options, giving an estimate of the true subjective difference between the two items.

- Study 4 (Decisions from Description / DFD): Participants chose between pairs of monetary lotteries, 
where each lottery had three probabilistic outcomes (e.g., $30 \%$ chance of $\$ 15,45 \%$ chance of $\$ 50,25 \%$ chance of \$77).

- Study 5 (Posters): Participants decided which of two posters they preferred. As in the food study, they had previous rated the subjective quality of the two posters they saw.

- Study 6 (Decisions from Experience / DFE): Participants had to decide which of two decks of cards they would prefer to draw from for a monetary outcome. As opposed to the decisions from description, participants in this study learned about the outcomes of each deck by drawing cards from each one.

- Study 7 (Flash gambling task / FGT): Participants saw two patches of flashing dots that varied from frame to frame $(20 \mathrm{~Hz})$. The patches varied in terms of their mean and standard deviation of the number of dots on each frame, and participants had to decide which one had more dots on average, or which one they would prefer to draw from (their reward was based on a random draw from the chosen patch).

Studies 1, 6, and 7 were experiments in which participants received information piece by piece, allowing us to directly compare the information they saw against the information we would expect based on a random draw from the stimulus. Studies 2-5 were eye tracking studies, where we compared the value of stimulus attributes that participants looked at (observed) against the expected value of the stimuli that participants would have sampled if they looked randomly at stimulus attributes. More details on each study are provided in the methods and supplementary information, but these three characteristics allowed us to examine how the true information generated by the stimulus compared to the information that participants had at the end of the decision making process.

\section{Polarization}

We carried out two analyses to test for polarization, which compared the true stimulus information against the information that participants actually collected. Each of the analyses presented in this paper used a Bayesian method for estimation, with uninformative priors that allowed the data to supplant the priors in determining the results. Each analysis was implemented in JAGS (Plummer, 2003), using MCMC sampling to estimate the posterior distribution of the statistic of interest (variance ratio, correlation, difference, etc.). For all analyses, we carried out 4 chains of 5000 samples each, checked for convergence, and computed the $95 \%$ highest density interval (HDI) for each effect (see Kruschke, 2014). More detail on these methods are provided in the methods and supplementary material.
The first of these compared the expected variance of the stimulus information against the true variance of the information collected by participants (variance ratio). Across six out of the seven studies, we found that the variance in the information gathered by participants was greater than would be expected given a representative sample from the distribution (Figure 2). The variance ratio for each experiment is provided in the first column of Table 1: Variance ratios greater than one indicate polarization, where the observed distribution of information is larger than the expected variance of a representative sample from the stimulus.

The second analysis computed a difference score between the observed information gathered by participants and a random draw from the stimulus based on the same number of pieces of information. For this analysis, we took a random draw from the stimulus distribution for each piece of information participants drew, then compared the average value of these random draws to the average value of the information that participants observed by taking the difference between their means. If the participant's draws were further from the stimulus mean than the random draw, this difference was positive; if the participant's draw was nearer to the stimulus mean, this difference was negative. The result for each experiment is shown in the second column of Table 1: for five out of the seven studies, the overall mean difference was positive, indicating that participants had drawn information on each trial that was more extreme than a random draw from the stimulus.

The patterns of polarization in these studies are also shown in Figure 2. The expected distribution of information that we would anticipate from a representative sample is shown in orange, while the observed distribution of information collected by participants is shown as a blue histogram. In nearly all cases, it is clear that the histogram shows a more widely dispersed pattern of information than the expected distribution.

The only study that showed a pattern of results that was inconsistent with a pattern of polarization, as opposed to being inconclusive, was the Flash Gambling Task (Pleskac et al., 2019) study, and the inconsistency was observed on the variance ratio measure. Because information arrived rapidly in this study (the screen updated at $20 \mathrm{~Hz}$ ), participants were likely unable to consider every single piece of information. Furthermore, even if participants had made up their minds after gathering an extreme piece of information, information arrived continuously until they made their response. If the intervening period were around 200-300 ms (typical nondecision times for a perceptual binary choice task, Ratcliff \& McKoon, 2008), then they would have gathered at least 4-6 additional samples of evidence, diluting the extremity of the pool of information they appear to have considered before the end of the trial. This would naturally wash out any effects of the participants' stopping rules and negate the 


\section{Table 1}

Table of mean effects [95\% HDIs] related to polarization and extremism for each of the seven studies. The variance ratio is the relative variance of the observed versus expected samples (greater than 1 indicates a polarized distribution). Extremity is the difference in extremeness between observed samples and randomly drawn samples from the expected distribution (positive values indicate polarization). For extremists, we examined the relationship between the extremeness of evidence on a particular trial and the amount of information gathered on that trial (Have less information), the response times on those trials (Decide faster), and the threshold estimates from a diffusion model for the corresponding participant, where available (Lower thresholds)

\begin{tabular}{l|cc|ccc}
\multirow{2}{*}{ Study } & \multicolumn{2}{|c}{ Polarization } & \multicolumn{3}{c}{ Extremists } \\
\hline Dots & $1.21[1.16,1.26]$ & $0.01[0.00,0.01]$ & $-.39[-.42,-.36]$ & $-.41[-.44,-.39]$ & Lower thresholds \\
Currency & $7.24[6.86,7.69]$ & $0.22[0.13,0.30]$ & $-.11[-.15,-.07]$ & $-.07[-.11,-.03]$ & $.01[-0.03,0.04]$ \\
Food & $3.21[3.08,3.36]$ & $-0.03[-0.10,0.04]$ & $-.04[-.07,-.01]$ & $-.14[-.17,-.10]$ & $-.04[-.05,-.03]$ \\
DFD & $3.28[3.09,3.51]$ & $-0.10[-0.73,0.48]$ & $-.10[-.15,-.05]$ & $-.14[-.19,-.10]$ & $-.08[-.11,-.06]$ \\
Posters & $8.51[8.09,9.98]$ & $0.40[0.32,0.48]$ & $-.04[-.08,-.00]$ & $-.17[-.20,-.13]$ & $-.06[-.08,-.04]$ \\
DFE & $2.13[2.10,2.17]$ & $19.65[17.78,21.34]$ & $-.19[-.20,-.17]$ & $-.23[-.24,-.21]$ & \\
FGT & $0.78[0.77,0.79]$ & $0.17[0.04,0.29]$ & $-.25[-.26,-.24]$ & $-.55[-.56,-.55]$ & $-.04[-.05,-.03]$
\end{tabular}
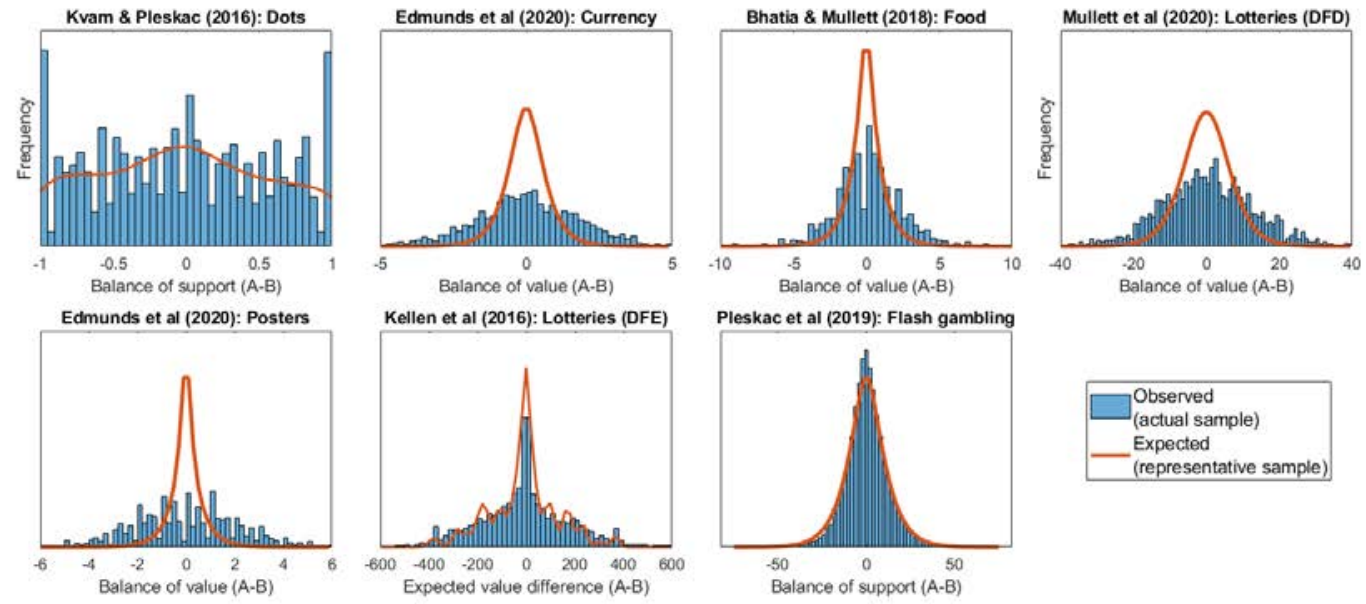

Figure 2

Distribution of observed information gathered by participants (blue histograms) compared to the expected distribution of information that a participant should have, given representative draws from the true distributions.

typical polarization effect that we observed in other studies.

\section{Extremists in the distribution}

Although the presence of polarization is an important implication of widespread use of optimal choice strategies, it is not the only interesting phenomenon that arises as a consequence of implementing these strategies to make decisions. Among populations of decision makers, there will naturally be variation in the value that people place on making the correct (accurate, informed) decision versus making a timely decision. This trade-off between speed and accuracy is reflected in the setting of the threshold (Heitz, 2014; Reed, 1973; Wickelgren, 1977). As illustrated in the proof and simulations in the supplementary material, a lower threshold results in information with a more variable mean due to the properties of small samples. The decision makers at the poles of the distribution tend to make up their minds after seeing only a few pieces of extreme information.

We refer to these outlying decision makers as extremists, as they tend to be at the tails of the distributions of information and possess extremely distorted views of the information that is in their environments. But how are these individuals created, given a group of decision makers that are all implementing optimal strategies? The answer lies in the combination of stopping rules implemented by the decision makers, and the variability in information that decision makers can sample from their environments. Extremism appears when a decision maker who favors fast decisions (low thresholds) encounters a piece (or small set) of extreme information. This piece of information allows them to immediately cross 
their threshold and favor $A \succ B$ or $B \succ A$. They can therefore stop gathering new information, meaning that the extreme view provided by the lone piece of extreme information is never combined or tempered with conflicting evidence.

To characterize extremism using the empirical data from the seven studies, we ran three main analyses. The first looked at the relationship between extremism and the amount of information gathered by decision makers by simply correlating the average strength of information gathered on each trial with the total number of pieces of information. The results are shown in the third column of Table 1. As we might expect, smaller samples tend to be more volatile, and thus the extremists are likely to have less information than their peers. In all seven studies, this is evidenced by a substantial negative correlation between the number of pieces of information and the overall extremity of that information.

The second observation characterizing extremists was that they tended to make quicker decisions than their peers. Decision speed is typically related to but distinguishable from the number of samples drawn by participants - a person could very carefully consider a few pieces of information and reach a decision in the same amount of time that another person gathers and considers a large volume of information. The relationship between decision time and evidence extremity is shown in the fourth column of 1 . As before, we computed a simple Bayesian linear correlation between the average strength of information collected on each trial of the study with the response time on that trial. Also, as before, all seven studies showed a negative relationship between response time and information extremity, suggesting that participants with extreme views tended to make faster decisions during the experiments.

The final observation about extremists involves characterizing them in terms of an underlying mechanism, such as determining what made these particular individuals become extremists. We suggest that low thresholds, indicating a preference for speedy as opposed to well-informed decisions, is more common among decision makers who wound up on the fringes. To investigate this, we applied a simple diffusion model (Ratcliff et al., 2016; Wagenmakers et al., 2007) to predict choices and response times in terms of the strength of information favoring one option over another (drift), priority for speedy or accuracy (threshold), and the length of time spent on processes unrelated to choice such as encoding stimuli and making a response (non-decision time). For each trial of each study, the drift rate was fixed based on the true average difference in value between stimuli. Threshold and non-decision time were permitted to vary across participants, so that each person was assigned a unique value for their threshold. This allowed us to compare the average extremeness of an individual participant's responses (extremism) against a rough estimate of the threshold they used to make choices.
This analysis was carried out for only five of the studies, as the dots study contained a manipulation that artificially induced a relationship between the amount of information and speed of decision making (a manipulation of weight Kvam \& Pleskac, 2016) and the DFE study has a discrete structure that means response times are not commensurate with the number of pieces of information drawn. ${ }^{1}$ Of the remaining five studies, four of them showed a substantive negative correlation between extremism and thresholds (rightmost column of Table 1), indicating that decision makers with more extreme views did tend to favor fast decisions over well-informed ones.

Put together, we can characterize decision makers with more extreme views as ones who gather very little information, who make decisions quickly, and who are impatient (value time over rigor, leading to lower thresholds). But these extremists may be the product of people reacting rationally to choice incentives, and are not necessarily intentionally malicious actors. Rational decision making processes - resulting from dual pressures on decision quality and time - therefore create polarized groups of decision makers as well as naturally generate a subset of uninformed individuals who hold extreme views.

\section{Discussion}

Even in simple perceptual tasks, where there should be little motivation to distort information or engage in biased information processing, we observed multiple cases where the information people gathered was more extreme than a representative sample from their environment should be. Critically, motivated information search is not necessary to account for these results, although predecision or information search biases would exacerbate the issues we outline here. In the supplemental material, we examine in more depth how thresholds, accumulation biases (motivated information search), and predecision biases (viewpoints held before any information is gathered) affect the profiles of information decision makers will have collected following a choice. Our simulations show that low thresholds and high predecision bias in particular will exacerbate polarization and encourage extremism, but the mechanism that ultimately creates polarization and extremism is the two-boundary decision process.

Due to the ubiquity of decision making, it is possible that any social information that we get from other people is biased toward extremes. As opposed to information we gather from non-social sources, social information has potentially been passed through a "decision filter" that effectively removes moderate positions that would be occupied by people who

\footnotetext{
${ }^{1}$ It is also worth noting that a diffusion model has never been applied to decisions from experience, to the authors' knowledge. Its suitability for modeling response times on this kind of task has therefore not already been established.
} 
have a roughly even balance of information between choice options. The lack of moderates in the population of decision makers following choice is juxtaposed against the prevalence of extremists, who hold particularly biased information compared to the true information in their environments. Our analyses showed that the more extreme decision makers were also the least-informed, quickest to decide, and the the most impatient (lower thresholds). As we show in the supplementary information, extremists tend to show up most when there is time pressure to make choices and when decision makers begin the choice process with a biased position; but they are nonetheless created through a rational decision process.

A major concern regarding these extremists is the degree of influence that they can carry over others. Despite having relatively little information, these decision makers are likely to believe that their views are representative of the true information in their environment, referred to as the "law of small numbers" (Tversky \& Kahneman, 1971; Rabin, 2002). This suggests that extremists will be confident in their views and willing to spread them, despite the information they have gathered possessing relatively poor reliability. This is exacerbated by the fact that extremists will be the first to share the information they have - by virtue of taking less time to make decisions, they are free to share their views before those who have taken more time and carefully constructed their beliefs with large samples of information. Extremists will be the first to influence their peers via word of mouth and social networks (Lynn et al., 2017), provide reviews of new products that influence many subsequent buyer decisions (Stephen \& Lehmann, 2016), and spread hateful views that carry quickly through social networks (Mathew et al., 2019). Social media algorithms base recommendations on early posters, meaning that they will carry greater weight in determining subsequent users' views and activity as well (Bastide et al., 2018; Lynn et al., 2017).

To add insult to injury, these extremists will also carry greater weight in cultural discourse and the formation of public opinion. Those with the most extreme positions carry a disproportionately greater weight over the course of information transmission between individuals simply by being extreme (Navarro et al., 2018). Furthermore, decision makers with small, extreme samples of information are still confident in their views (Kvam \& Pleskac, 2016; Griffin \& Tversky, 1992), possibly due to a belief in the "law of small numbers" where people think even very small samples will possess the same statistical properties as large samples (Tversky \& Kahneman, 1971). As a result, we arrive at the rather unfortunate scenario where the least-informed (fewest samples of information) and most impatient (lowest-threshold) individuals in a population will have the greatest influence on public opinion. Perhaps these basic decision processes can explain why perceived political polarization is exaggerated-people tend to think that the political left and right in the US are far more polarized than they are (Westfall et al., 2015).

\section{Alleviating the problem}

Despite the relatively bleak outlook of our results, where uninformed and impulsive extremists control the cultural narrative around topics on which we make decisions, we are not doomed to polarization and extremism in all scenarios. Strategies that are non-optimal for reward rate maximization, but satisfy other criteria, can be useful for reducing polarization among groups of people. For example, a strategy elicited by incentivizing estimation accuracy - where a person is asked to estimate how much the information in their environment favors one option or another - can create a distribution of information that is representative of the true information in the environment. This kind of task can be accomplished by setting a goal for how far from the true mean of the underlying distribution the agent is willing to be. In this approach, a decision maker estimates the mean of the distribution when the error of their accumulated samples falls below a certain level.

This type of strategy is illustrated in Figure 3: as opposed to decisions where the decision maker samples information until they shift sufficiently far toward one end or another, a goal of precision will encourage the decision maker to continue gathering new information, shifting their estimate over time (blue line) until the error of the mean (shaded blue area) falls below a criterion level. Once their estimate is precise enough, a decision maker can safely make a claim about the location of the mean. Thus, if estimation is the goal, as opposed to a decision, then the distribution of information across a population of decision makers (right side of Figure 3) will correspond closely to the input distribution (left side of Figure 3). Posing the task as an estimation problem can therefore encourage participants to stop when they have a precise (low error, internally consistent) pool of information rather than a strong (extremely favoring one option over another) pool of information (Kvam \& Pleskac, 2016). With the goal of estimation, decision makers become judges, and the resulting distribution of information among them will be unbiased and concentrated around the true mean of the information in their environments.

In the political sphere, our results suggest that binary or multiple-choice voting - requiring voters to decide in favor of only Candidate A or Candidate B (or C, D, E, etc) - may be partly responsible for the current state of political polarization. It may be possible to alleviate political polarization through approaches like cardinal voting (Baujard et al., 2018), which incentivizes precise ratings (estimation) and allows ties, as opposed to forcing voters to select one option over another. Similar interventions could reduce polarization on social issues-rather than voting yes or no on gay marriage, legalization of marijuana, abortion, and climate change, voters should be encouraged to judge the merits of arguments for 

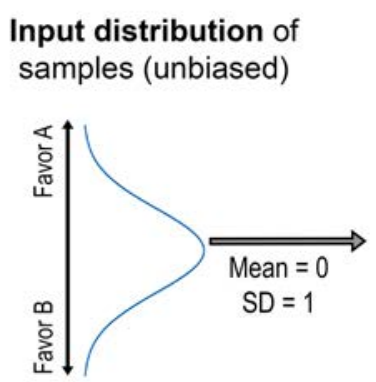

Estimation / judgment process (until desired precision)
Final distribution of sampled information (unbiased)

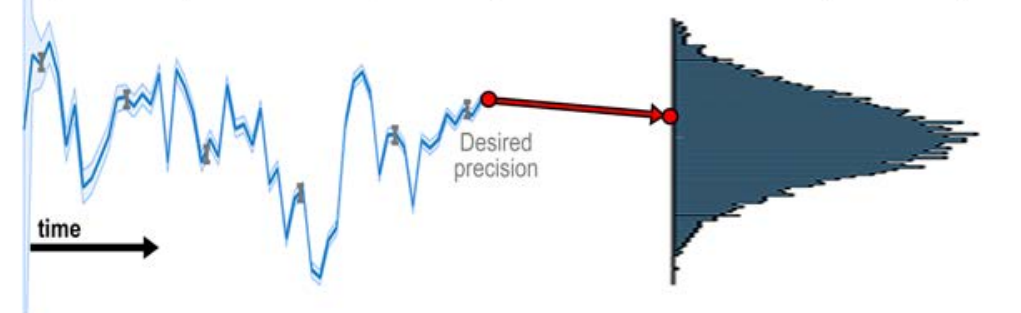

Figure 3

Illustration of a precision-based judgment process, where the goal is not to select one side over the other (as in Figure 1) but to meet a criterion level of estimation precision.

or against, or to rank their choices for, a variety of solutions to these complex topics.

Another way to reduce polarization is to simply force decision makers to take some number $n$ pieces of information before stopping, as in fixed-sampling paradigms (Busemeyer, 1985; Fried \& Peterson, 1969). If the information they gather is independent and identically distributed (i.i.d.) across samples, as is typically assumed, decision makers will obtain a representative sample of the true information in the environment and thus polarization will not occur. However, this approach is paternalistic and thus potentially very difficult to implement, as it takes the autonomy of information collection away from decision makers (causing people's well-being and performance to suffer Deci \& Ryan, 1995).

\section{Conclusions}

In a decision scenario that incentivizes a trade-off between time and decision quality, a population of rational decision makers will become polarized. In this paper, we have shown this through simulations, a mathematical proof (supplementary materials) and demonstrated it empirically in seven studies. This leads us to an unfortunate but unavoidable conclusion that decision making is a bias-inducing process by which participants gather representative information from their environment and, through the decision rules they implement, distort it toward the extremes. Such a process also generates extremists, who hold extreme views and carry undue influence over cultural discourse (Navarro et al., 2018) despite being relatively uninformed and impulsive (low thresholds; Kim \& Lee, 2011). We have suggested several avenues for interventions, foremost among them providing incentives favoring estimation or judgments as opposed to incentives for timely decision making. Our hope is that future work testing and implementing these interventions will reduce the prevalence of polarization and extremism across social domains currently occupied by decision makers.

\section{References}

Ahler, D. J., \& Sood, G. (2018). The parties in our heads: Misperceptions about party composition and their consequences. The Journal of Politics, 80(3), 964-981.

Bail, C. A., Argyle, L. P., Brown, T. W., Bumpus, J. P., Chen, H., Hunzaker, M. F., ... Volfovsky, A. (2018). Exposure to opposing views on social media can increase political polarization. Proceedings of the National Academy of Sciences, 115(37), 9216-9221.

Baldassarri, D., \& Gelman, A. (2008). Partisans without constraint: Political polarization and trends in american public opinion. American Journal of Sociology, 114(2), 408-446.

Bastide, P. R., Deluca, L. S., \& Do, L. M. (2018, January 16). User recommendations in a social media network. Google Patents. (US Patent 9,871,758)

Baujard, A., Gavrel, F., Igersheim, H., Laslier, J.-F., \& Lebon, I. (2018). How voters use grade scales in evaluative voting. European Journal of Political Economy, 55, $14-28$.

Benkler, Y., Faris, R., \& Roberts, H. (2018). Network propaganda: Manipulation, disinformation, and radicalization in american politics. Oxford University Press.

Bogacz, R., Brown, E., Moehlis, J., Holmes, P., \& Cohen, J. D. (2006). The physics of optimal decision making: A formal analysis of models of performance in twoalternative forced-choice tasks. Psychological Review, 113(4), 700-765. doi: 10.1037/0033-295X.113.4.700

Bogacz, R., Wagenmakers, E.-J., Forstmann, B. U., \& Nieuwenhuis, S. (2010). The neural basis of the speed-accuracy tradeoff. Trends in Neurosciences, 33(1), 10-16. doi: 10.1016/j.tins.2009.09.002 
Brady, W. J., Wills, J. A., Jost, J. T., Tucker, J. A., \& Van Bavel, J. J. (2017). Emotion shapes the diffusion of moralized content in social networks. Proceedings of the National Academy of Sciences, 114(28), 7313-7318.

Busemeyer, J. R. (1985). Decision making under uncertainty: a comparison of simple scalability, fixed-sample, and sequential-sampling models. Journal of Experimental Psychology: Learning, Memory, and Cognition, 11(3), 538.

Busemeyer, J. R., Gluth, S., Rieskamp, J., \& Turner, B. M. (2019). Cognitive and neural bases of multi-attribute, multi-alternative, value-based decisions. Trends in cognitive sciences, 23(3), 251-263.

Coenen, A., \& Gureckis, T. (2016). The distorting effect of deciding to stop sampling. In A. Papafragou, D. Grodner, D. Mirman, \& J. Trueswell (Eds.), Proceedings of the 37th Annual Meeting of the Cognitive Science Society (pp. 2819-2824).

Crockett, M. J. (2017). Moral outrage in the digital age. Nature Human Behaviour, 1(11), 769-771.

Deci, E. L., \& Ryan, R. M. (1995). Human autonomy. In Efficacy, agency, and self-esteem (pp. 31-49). Springer.

DeKay, M. L. (2015). Predecisional information distortion and the self-fulfilling prophecy of early preferences in choice. Current Directions in Psychological Science, 24(5), 405-411.

DeKay, M. L., Patiño-Echeverri, D., \& Fischbeck, P. S. (2009). Distortion of probability and outcome information in risky decisions. Organizational Behavior and Human Decision Processes, 109(1), 79-92.

Edwards, W. (1965). Optimal strategies for seeking information: Models for statistics, choice reaction times, and human information processing. Journal of Mathematical Psychology, 2(2), 312-329. doi: 10.1016/0022-2496(65) 90007-6

Festinger, L. (1957). A theory of cognitive dissonance (Vol. 2). Stanford university press.

Foer, F. (2017). World without mind. Random House.

Fried, L. S., \& Peterson, C. R. (1969). Information seeking: Optional versus fixed stopping. Journal of Experimental Psychology, 80(3p1), 525.

Gold, J. I., \& Shadlen, M. N. (2007). The neural basis of decision making. Annual review of neuroscience, 30.

Griffin, D., \& Tversky, A. (1992). The weighing of evidence and the determinants of confidence. Cognitive psychology, 24(3), 411-435.
Heitz, R. P. (2014). The speed-accuracy tradeoff: history, physiology, methodology, and behavior. Frontiers in Neuroscience, $8,150$.

Heitz, R. P., \& Schall, J. D. (2012). Neural mechanisms of speed-accuracy tradeoff. Neuron, 76(3), 616-628. doi: 10.1016/j.neuron.2012.08.030

Hogg, M. A., \& Turner, J. C. (1985). Interpersonal attraction, social identification and psychological group formation. European journal of social psychology, 15(1), 5166.

Iyengar, S., Sood, G., \& Lelkes, Y. (2012). Affect, not ideologya social identity perspective on polarization. Public opinion quarterly, 76(3), 405-431.

Kim, S., \& Lee, D. (2011). Prefrontal cortex and impulsive decision making. Biological psychiatry, 69(12), 11401146.

Klein, E. (2020). Why we're polarized. Profile Books.

Kruschke, J. (2014). Doing bayesian data analysis: A tutorial with $R, J A G S$, and STAN. Academic Press.

Kvam, P. D., \& Pleskac, T. J. (2016). Strength and weight: The determinants of choice and confidence. Cognition, 152, 170-380. doi: 10.1016/j.cognition.2016.04.008

Lewis, M. (2008). Self-conscious emotions: Embarrassment, pride, shame, and guilt. In Handbook of emotions, $3 \mathrm{rd} \mathrm{ed}$ (pp. 742-756). New York, NY, US: The Guilford Press.

Lynn, T., Muzellec, L., Caemmerer, B., \& Turley, D. (2017). Social network sites: early adopters' personality and influence. Journal of Product \& Brand Management.

Mathew, B., Dutt, R., Goyal, P., \& Mukherjee, A. (2019). Spread of hate speech in online social media. In Proceedings of the 10th acm conference on web science (pp. 173182).

Messick, D. M., \& Mackie, D. M. (1989). Intergroup relations. Annual review of psychology, 40(1), 45-81.

Milgram, S., Sabini, J. E., \& Silver, M. E. (1992). The individual in a social world: Essays and experiments. Mcgraw-Hill Book Company.

Navarro, D. J., Perfors, A., Kary, A., Brown, S. D., \& Donkin, C. (2018). When extremists win: Cultural transmission via iterated learning when populations are heterogeneous. Cognitive science, 42(7), 2108-2149.

Nickerson, R. S. (1998). Confirmation bias: A ubiquitous phenomenon in many guises. Review of general psychology, 2(2), 175-220. 
Pleskac, T. J., Yu, S., Hopwood, C., \& Liu, T. (2019). Mechanisms of deliberation during preferential choice: Perspectives from computational modeling and individual differences. Decision, 6(1), 77-107.

Plummer, M. (2003). JAGS: A program for analysis of Bayesian graphical models using Gibbs sampling. In Proceedings of the 3rd international workshop on distributed statistical computing (Vol. 124, p. 10).

Rabin, M. (2002). Inference by believers in the law of small numbers. The Quarterly Journal of Economics, 117(3), $775-816$.

Ratcliff, R., \& McKoon, G. (2008). The diffusion decision model: Theory and data for two-choice decision tasks. Neural Computation, 20(4), 873-922. doi: 10.1162/ neco.2008.12-06-420

Ratcliff, R., Smith, P. L., Brown, S. D., \& McKoon, G. (2016). Diffusion decision model: Current issues and history. Trends in cognitive sciences, 20(4), 260-281.

Reed, A. V. (1973). Speed-accuracy trade-off in recognition memory. Science, 181(4099), 574-576.

Stephen, A. T., \& Lehmann, D. R. (2016). How wordof-mouth transmission encouragement affects consumers' transmission decisions, receiver selection, and diffusion speed. International Journal of Research in Marketing, 33(4), 755-766.

Suhay, E. (2015, March). Explaining Group Influence: The Role of Identity and Emotion in Political Conformity and Polarization. Political Behavior, 37(1), 221-251. Retrieved 2020-10-09, from https://doi.org/10.1007/ s11109-014-9269-1 doi: 10.1007/s11109-014-9269-1

Taber, C. S., Cann, D., \& Kucsova, S. (2009). The motivated processing of political arguments. Political Behavior, 31(2), 137-155.

Tajfel, H., \& Turner, J. C. (1986). An integrative theory of group conflict. The social psychology of intergroup relations, 7-24.
Tajfel, H. E. (1978). Differentiation between social groups: Studies in the social psychology of intergroup relations. Academic Press.

Tversky, A., \& Kahneman, D. (1971). Belief in the law of small numbers. Psychological bulletin, 76(2), 105.

Wagenmakers, E.-J., Van Der Maas, H. L. J., \& Grasman, R. P. P. P. (2007). An EZ-diffusion model for response time and accuracy. Psychonomic bulletin \& review, 14(1), 3-22.

Wald, A., \& Wolfowitz, J. (1949). Bayes solutions of sequential decision problems. Proceedings of the National Academy of Sciences of the United States of America, 35(2), 99-102.

Westerwick, A., Johnson, B. K., \& Knobloch-Westerwick, S. (2017). Confirmation biases in selective exposure to political online information: Source bias vs. content bias. Communication Monographs, 84(3), 343-364.

Westfall, J., Van Boven, L., Chambers, J. R., \& Judd, C. M. (2015, March). Perceiving Political Polarization in the United States: Party Identity Strength and Attitude Extremity Exacerbate the Perceived Partisan Divide. Perspectives on Psychological Science, 10(2), 145-158. Retrieved 2020-09-07, from https://doi.org/10.1177/ 1745691615569849 (Publisher: SAGE Publications Inc) doi: $10.1177 / 1745691615569849$

Wickelgren, W. A. (1977). Speed-accuracy tradeoff and information processing dynamics. Acta Psychologica, 41(1), 67-85. doi: 10.1016/0001-6918(77)90012-9

Wilson, A. E., Parker, V., \& Feinberg, M. (2020). Polarization in the contemporary political and media landscape. Current Opinion in Behavioral Sciences, 34, 223-228. 
Supplemental material for "Polarization and extremism emerge from rational choice"

Peter D. Kvam

University of Florida

Matthew Baldwin

University of Florida 
Supplemental material for "Polarization and extremism emerge from rational choice"

In this document, we provide a proof giving the expected distribution of information among decision makers, present a brief overview of the methods from each of the studies we present in the main text, provide additional details on the analyses we ran, and examine simulations based on manipulations of the decision making process. We additionally show that our results will hold for multi-alternative choice, such that polarization and extremism are not confined strictly to the domain of binary choice.

\section{Proof - expected distributions of evidence}

Consider a choice scenario where a decision maker has two options they could select. They make their decision by gathering new pieces of information, which they use to sequentially update their beliefs about a stimulus. In order to achieve a particular level of accuracy while minimizing response times, the optimal strategy is to track the balance of support $s$ for option A versus option $\mathrm{B}$, and stop when this balance exceeds a threshold value $\theta$. Each new piece of information can increase or decrease the balance of support. Thus, the optimal decision maker gathers new information piece by piece until either $s \geq \theta$ or $s \leq-\theta$ (Edwards, 1965; Bogacz et al., 2006).

Suppose that new information is sampled from a true underlying distribution $f(x)$ that defines a distribution of evidence values it could take on $-\infty<x<\infty$ such that $\int_{-\infty}^{\infty} f(x)=1$. The function $F(x)$ is the cumulative distribution function, determining the probability that the value of a new piece of information $x_{1}$ is less than or equal to $x$.

For now, we assume that all decision makers start in an unbiased state represented by the random variable $s(t)$, such that $s(0)=0 .{ }^{1}$ Our goal is to derive the distribution of evidence states, specified by a probability density $g(x)$ over evidence levels $x$, that will result from a population of decision makers starting at evidence state $s_{0}=0$ and sampling new information from $f(x)$ until they reach state $\pm \theta$. We will designate the distribution of states of decision makers who finish at time $t$ as $g_{t}(x)$, and the states of decision makers who have not finished before time $t$ as a random

\footnotetext{
${ }^{1}$ Variations on this assumption are explored in the model simulation section.
} 
variable $s(t)$.

The likelihood that a decision maker will stop after sampling a single piece of information $x_{1}$, drawn randomly as $x_{1} \sim f(x)$, is simply the probability of sampling a piece of information that moves the decision maker past one of the thresholds, $\operatorname{Pr}(t=1)=F(-\theta)+(1-F(\theta))$. The distribution of evidence among those decision makers who have enough support to stop after this first piece of information, $g_{1}(x)$, will be

$$
g_{1}(x)=f(x \mid x \leq-\theta)+f(x \mid x \geq \theta)
$$

Meanwhile, those decision makers who do not stop after a single piece of evidence will be distributed between $-\theta$ and $\theta$. The "unfinished" states at time point 1 will be $s_{1}$, distributed according to a truncated distribution of $f(x)$ :

$$
\operatorname{Pr}\left(s_{1}=x\right) \sim \frac{f(x \mid-\theta<x<\theta)}{1-\operatorname{Pr}(t=1)}
$$

Those decision makers who have not made a selection yet will have to sample another piece of information from the source distribution $f(x)$, giving $s_{1}+x_{2}$ where $x_{2}$ is a random variable drawn from $f(x)$. Some of these decision makers will then be able to stop and make a choice, if they have passed the threshold. Thus, the distribution of decision makers who have enough support to stop after two pieces of information will be

$$
g_{2}(x)=\operatorname{Pr}_{x}\left(x=s_{1}+x_{2}\right) \cdot[\operatorname{Pr}(x \leq-\theta)+\operatorname{Pr}(x \geq \theta)]
$$

where $x_{2} \sim f(x)$. This procedure of adding a random variable from $f(x)$ to the existing distribution of states $s_{n}$, calculating the distribution below $-\theta$ / above $\theta$, and truncating the remaining distribution of states can be carried out ad infinitum to get the distribution over evidence states that finish at each time point $n$.

$$
g_{n}(x)=\operatorname{Pr}_{x}\left(x=s_{n-1}+x_{n}\right) \cdot[\operatorname{Pr}(x \leq-\theta)+\operatorname{Pr}(x \geq \theta)]
$$


Put together, this specifies a series of functions $g_{1}(x), g_{2}(x), \ldots, g_{n}(x)$ that specify the distribution of evidence among decision makers that stop after the first, second, ..., $n^{\text {th }}$ piece of information. Importantly, the probability of stopping on a particular step $t$, given that the participant has not stopped yet, is given by the integral of $g_{n}(x)$ :

$$
\operatorname{Pr}(t=n \mid t>[n-1])=\int_{x=-\infty}^{\infty} g_{n}(x) d x .
$$

Therefore, we can calculate the cumulative probability of stopping at or before step $t$ as $G(t)=\operatorname{Pr}(t=n \mid t>[n-1]) \cdot(1-G(t-1))$, noting that $G(1)=\int g_{1}(x) d x$ and calculating subsequent values iteratively. Because $s(t)$ follows a Wiener process, there are well-established methods to determine $g(t)$ and $G(t)$ (see Diederich \& Busemeyer, 2003; Ratcliff, 1978). The overall distribution of evidence across decision makers at the conclusion of the decision process $S_{t}(x)$ can be then computed as

$$
S_{t}(x)=\sum_{t=1}^{\infty} g_{t}(x) \cdot(1-G(t))
$$

We could use $S_{t}(x)$ as the final distribution of evidence among decision makers who have finished deciding. However, it seems to make more sense for a decision maker's opinion to be determined by the average of all of the pieces of information they have collected (Turner et al., 2017), rather than as the sum of these pieces of information. ${ }^{2}$ The distribution of average evidence for decision makers arriving at a choice at time $t$ is computed by simply mapping $g_{t}(x) \rightarrow g_{t}\left(\frac{x}{t}\right)$, stretching or shrinking the $\mathrm{x}$-scale by the number of pieces of information that had been drawn at time $t$.

\section{Methods}

Each of the studies we analyzed came from an open data set hosted on the Open Science Framework (OSF). The dots data are provided at osf.io/ba5c7; the currency, food, poster, and

\footnotetext{
${ }^{2}$ We examine the latter approach below in our simulations, but the summing rule makes the somewhat unreasonable prediction that there will be no decision makers whose beliefs lie between $-\theta$ and $\theta$
} 
lottery / DFD data are provided at osf.io/mvk95 (provided in association with Edmunds et al., 2020a); the decisions from experience / DFE data are provided at osf.io/ngc45; and the flash gambling task data (Study 1) are provided at osf.io/g7a49. These studies were chosen so that we knew the data were (1) coming from an incentivized decision making task, (2) tracked the information that participants considered during the decision process, and (3) had all of the necessary information to compute the expected mean and variance of evidence of a random sample for the stimuli on each trial. We summarize the essential characteristics of the methods from each study here, but more detailed descriptions and explanations can be found in the original papers.

Study 1 - Dots (Kvam \& Pleskac, 2016). In the dots study, participants were presented with a series of dots that appeared on the screen and asked to decide whether the dots were being pulled from a pool of dots that was $2 / 3$ green and $1 / 3$ pink or $2 / 3$ pink and $1 / 3$ green. From trial to trial, the proportion of dots that were actually pink or green (strength) was manipulated, as well as the rate at which these dots were presented (weight). Note that this presented a confound for threshold analyses, as thresholds in the original study were found to increase with higher values of strength and weight manipulations (Kvam \& Pleskac, 2016), creating a violation of selective influence that would artificially increase the correlation between threshold estimates and the amount of evidence that participants gathered. This study included a total of 29 participants who each completed $\sim 200$ trials of the task.

Study 2 - Currency (Edmunds et al., 2020b). In this study, participants saw three currencies for each of two response options. Their task was to pick the set of three currencies that had the higher overall value (i.e., would yield the greatest total value sum). The currencies varied in type, including yen, pounds, and Q (made-up currency), and participants were given the exchange rates of each of these currencies to US dollars. Participants' gaze was tracked during the study so as to examine which of the outcomes (which of the six currencies) they were considering on each fixation. Positive and negative "sides" were randomly assigned such that the currencies on the top (bottom) conferred positive value while the currencies on the bottom (top) conferred 
negative value. This allowed us to examine the overall value of the pair of options: more positive indicated that the top (bottom) had greater value, while more negative indicated that the bottom (top) had greater value. On half the trials, the currencies displayed during a trial were all different (all three of yen, Q, and pounds for both options), and on the other half, the currencies displayed on a trial were all the same. We collapse across conditions for the analyses performed in this paper because they are not relevant to our research questions. Participants responded by pressing one key (up) to select the first option on the screen, or another key (down) to select the second option. This study included a total of 46 participants, who each completed 84 trials of the task.

Study 3 - Food (Bhatia \& Mullett, 2018). In the food study, participants saw two snack foods displayed on either side of the screen and were instructed to decide which one they preferred. Before the decision trials, participants rated each of the 50 snack foods that could be displayed as stimuli in the experiments on a 1-9 scale in terms of desirability. These desirability values were used as inputs for the analyses we used in this paper - for example, a food with desirability value of 4 vs one with a desirability value of 6 would have a value difference of 2 . As in the currency study, fixations were tracked and recorded. To get sample values from the eye tracking data, the desirability values of each option were centered so that fixating on the option with desirability 4 would have a value of -1 , and fixating on the option with desirability of 6 would have a value of +1 . A "representative" sample in this study was generated by randomly drawing the rated value of one snack or the other (50-50 chance). A total of 41 participants participated in this study, and each participant completed 100 trials.

Study 4 - Lotteries (Mullett et al., in prep). In the lotteries study, also referred to as a decisions from description study, participants were tasked with making decisions between two gambles. Each gamble had three equally likely outcomes, one each in the 10-30, 40-60, and 70-90 range. As in the currency study, values on the top / bottom were assigned positive / negative values so that positive outcomes for one option were contrasted against positive outcomes for the other option, providing a net expected value between choice options and allowing us to evaluate the variance of the values sampled on each fixation. A total of 54 participants completed 100 
trials each of this study.

Study 5 - Posters (Edmunds et al., 2020b). The posters study was similar to the food study, except that Likert scales were gathered after the choice task rather than before. A "representative" sample in this study was generated by randomly drawing the rated value of one poster or the other (50-50 chance). A total of 53 participants completed the task and had usable data, and each one completed 100 trials of the study.

Study 6 - Decisions from experience (Kellen et al., 2016). In the decisions from experience study, participants were tasked with both learning about and choosing between two gambles. In contrast to the decisions from description study, participants had to learn about each of the gambles by sampling from each one, rather than viewing the probabilities directly. For example, they could experience a series of samples $[\$ 4, \$ 0, \$ 4, \$ 4, \$ 0, \$ 4]$ from a gamble that was $75 \%$ chance of $\$ 4$ and $25 \%$ chance of $\$ 0$. The different choice problems varied in terms of the number, magnitude, and probability of the different outcomes that participants could receive from either choice option. Once participants indicated they were finished sampling, they chose one option or the other. The exact sequence of draws obtained by a participant in this task was recorded and used for our analyses - as in the other studies, one option was coded as negative and the other as positive in order to determine the expected and observed variance / mean of the samples that participants drew. A "representative" sample in this study was generated by drawing randomly between the available decks (50-50 chance) and generating outcomes according to their objective probabilities. A total of 104 participants completed the study, and each one completed 228 trials.

Study 7 - Flash gambling (Pleskac et al., 2019). In the flash gambling study, participants were tasked with determining which of two flashing patches of dots had a greater mean number of dots (inference) or which one they would prefer to take a random draw from, given that more dots meant a greater payoff (preference). One patch of dots had a constant number of dots (130), and the dots simply changed positions from frame to frame. For the other patch of dots, a random number was generated with a known mean (e.g., 140) and standard deviation (e.g., 20) on each 
frame, and the resulting number of dots was displayed. The difference between the certain and uncertain patches therefore followed a normal distribution, which was used to define a "representative" draw for our analyses. However, the patches were refreshed at $60 \mathrm{~Hz}$, making it very difficult to stop the stimulus immediately after obtaining a streak of information favoring one option or another. As a result, this study showed rather weak polarization results (see main text Figure 2, Table 1). A total of 113 participants each completed 960 trials of the experiment.

Put together, there were a total of 440 participants and over 150,000 trials across these data sets. They spanned inferential and preferential choice, eye tracking and sampling paradigms for gathering information, and both description and experience-based types of information. This wide-ranging set of studies suggests that the phenomena we observe are pervasive, and in particular that polarization and the presence of extremists are consequences of adopting relative-evidence decision strategies regardless of the particular domain of choice.

\section{Polarization analyses}

There were five main analyses carried out in the main text, whose results are presented in Table 1. For the variance ratio analyses, we compared the variance of the observed information collected by participants against the expected variance of information that participants would have collected from a purely random sample (i.e., whose size did not depend on the stopping rule during choice). For Studies 1, 6, and 7, this was trivial: the exact stimulus values that could be generated on each trial were known, so we could use the objective variance on each trial along with the number of samples drawn on each trial to calculated the expected variance of the mean of the sample if it were representative. For these studies, the stimuli either generated multinomial random variables or normal random variables on each draw. Thus, we could calculate the expected variance of the mean of the multinomial / normal random variable for a given number of samples (given by the number the participant drew). This was calculated for every trial of every study, giving us a distribution of expected variance of the stimuli against which we could compare the observed variance of participants' samples. 
As we might expect, the variance of observed and expected samples were generally quite close for these studies (Variance ratio in the first column of main text Table 1: Dots, DFE, and FGT).

Variance for the eye tracking studies was computed by taking the expected mean and variance for each individual fixation. It is the average of the squared differences between the expected value $\mu$ and the $n$ attribute values $a$ :

$$
\sigma^{2}=\frac{1}{n} \sum_{i}^{n}\left(a_{i}-\mu\right)^{2}
$$

These were then used to calculated the expected value and variance of the mean for a trial, which was simply the sum of the the multinomial random variables describing the values we would expect them to sample on each fixation divided by the number of fixations. This allowed us to compute the expected variance of the mean for Studies 2-5 (Currency, Foood, DFD, and Posters), against which we compared the observed average difference across fixations.

In contrast to the Dots, DFE, and FGT studies, the eye tracking studies tended to show particularly extreme patterns of variance. This seems to be due to a tendency toward autocorrelation in fixations - participants will look at the same option for multiple fixations (each typically around $250 \mathrm{~ms}$ ), and tend to look at the option they are about to select for several fixations especially leading up to choice. This is referred to as the gaze cascade (Shimojo et al., 2003), where the entropy of fixations reduces dramatically, thus distorting the information they consider relative to an unbiased sampling distribution. This gaze pattern provides evidence for the use of the relative evidence (rational / optimal) strategies that underlie the pattern of polarization observed in the data (Mullett \& Stewart, 2016).

The second polarization analysis was carried out based on similar information, except that we generated a multinomial (or normal, where appropriate) random variable for every draw made by the participants. The average of all the multinomial (normal) random variables was computer for each trial to give the expected direction and magnitude of the samples for that trial. For the extremity analysis, the observed average evidence from each trial was compared against the 
expected evidence. The difference between the two was used for the extremity analysis. If the observed sample was further from zero than the expected sample, the extremity value for that trial was positive; otherwise, it was negative.

The extremity analysis wound up having slightly different results than the variance ratio analysis for two reasons: first, the observed and expected outcomes for each trial could be on opposing sides of zero (as opposed to comparing variance, where squared values are necessarily greater than zero). Second, the values of the expected / observed values were not squared before being compared. Therefore, large differences from zero and differences that were not in the expected direction (i.e., were negative when the expected was positive) did not carry the same degree in the two methods of analysis. It is not clear which analysis is more "correct," so we simply present both of them in the Results.

\section{Extremist analyses}

The analyses for extremism were considerably simpler than those for polarization. The degree of extremeness of the evidence participants gathered on a particular trial was defined as simply the absolute value of the evidence they collected on that trial minus the true mean of the stimulus on that trial. This extremeness was simply correlated with the number of samples gathered on each trial (Extremeness $\times$ amount of information, Column 3 of Table 1), the response time on each trial (Extremeness $\times$ response time, Column 4 of Table 1), or the estimated threshold for each trial (Extremeness $\times$ threshold, Column 5 of Table 1).

Each of these correlation analyses were performed using Bayesian methods. The values for extremeness, amount of information, response times, and thresholds were standardized so that the resulting correlations would be interpretable on a $[-1,1]$ scale. The prior for the correlation between variables was a uniform distribution $U(-1,1)$, and they were estimated using JAGS (Plummer, 2003). The posterior was generated using 4 chains of 5000 samples, with 500 burn-in steps in each chain and starting points generated randomly from the prior. These same settings were used to estimate the polarization effects as well, except that the variance ratio had a wide 
uniform prior $U(0,1000)$ and the extremity had a wide normal prior $N(0,1000)$. This allowed us to approximate the posterior distribution of each of the effects - the means and and 95\% Highest Density Intervals (95\% HDIs) of the effects are reported in Table 1.

\section{Simulations}

Although they validate our predictions related to both polarization and extremism, it is useful to generate predictions and characterize choices beyond the scope of empirical studies we present in the paper. We do so here with simulations, which permit us to explore the shape of distributions that manifest across many thousands of trials as a function of important parameters of the model.

The three most important parameters of a random walk / diffusion model related to distribution of evidence are the direction / rate of evidence accumulation (drift), the stopping rule (threshold), and the starting point bias (prior bias) (Ratcliff et al., 2016). We manipulate each of these parameters in our simulations in order to explore their effect on polarization and extremism. Drift describes the information that participants gather, both in terms of what the stimulus provides and in terms of what the participants seek out themselves. It denotes the strength of an average piece of information that a participant will sample during the information accumulation process. Bias in the drift rate, usually modeled as the addition of a fixed constant (Diederich \& Busemeyer, 2006), would reflect a decision maker's tendency to intentionally or unintentionally sample information that agrees with a particular position, including a confirmation bias in information seeking.

The second main parameter in our simulations, threshold, governs how much information a decision maker wishes to gather before they are certain enough of their position to make a choice. As we suggested in the main text, lower thresholds result in a decision maker basing their choices on fewer pieces of information, because they are willing to stop sooner. Classic manipulations of thresholds include incentives encouraging fast (low threshold) or accurate (high threshold) responses, such as time pressure or harsh punishments for incorrect answers (Heitz, 2014; 
Wickelgren, 1977). As we suggested in the main text, lower thresholds tend to result in a greater incidence of extremism. The reason for this will become apparent in the simulations.

The final parameter that we manipulate in our simulations is one that has not been discussed at length thus far. Start point bias describes the beliefs or preferences that a decision maker has before they gather any information at all, meaning that a decision maker needs some reason to favor one response over the other in absence of any new information. This could occur naturally in political spheres, where a new policy can have biases for or against it due to the nature of people's views on the issue or the way it is associated with political positions. However, start point bias tends to appear less frequently in experiments where all the stimuli are new and/or randomly generated. The main way to manipulate start point biases is to incentivize responses on one side or another (for example, paying participants more if they respond "right" than "left"), or where participants get some predecision cue as to which option will be correct (Mulder et al., 2012).

The results of changing drift (accumulation bias), threshold, and start point (prior bias) are shown in Figure 1. We examine three main properties of the distributions: the proportion of responses favoring the right (+) or left (-) option, how widely spread decision makers are across the evidence space (width of the distributions) and the prevalence of extremists, which appear as "bumps" in the distributions far away from zero. Consider the top left panel, for example. There is an even distribution of responses favoring right and left alternatives (resulting from now drift bias), but responses are quite widely spread (high polarization) and there are substantial bumps in the distribution at around \pm 0.5 (indicating a high prevalence of extremists).

The top row of Figure 1 corresponds to cases where there is no drift bias and the information that participants gather or seek out does not systematically favor one option or the other, while the bottom row shows cases where participants systematically consider more information for the response option on the right (positive drift). From the simulations, it is clear that changes in drift actually have relatively minimal effects on the shape of the positive or negative sides of the distribution; instead, it simply determines what proportion of responses favor the left or the right option (choice proportions are provided on either side of each panel). This 

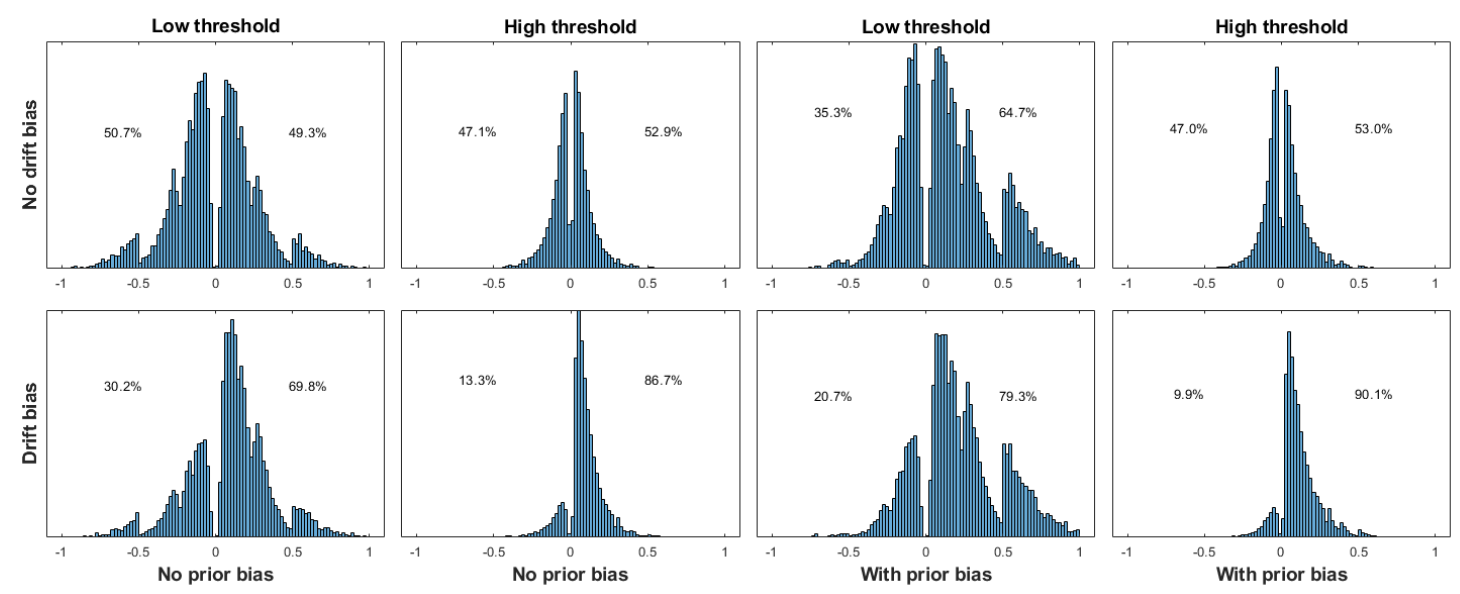

Figure 1. Distribution of evidence among populations of decision makers with varying drifts (top vs bottom), thresholds (colums $1 / 3$ vs $2 / 4$ ), and starting point biases (left vs right). Low thresholds tend to promote both polarization and extremism, while start point biases have a large effect on extremism in particular.

indicates that extremism is probably not the result of information search biases, because manipulating drift does not inherently increase the proportion of decision makers who occupy extreme positions - the bumps do not increase in size relative to the overall density of their respective side of the evidence distribution.

The first and third columns of Figure 1 correspond to cases where decision makers adopt a low threshold for their decisions, while the second and fourth columns correspond to cases where decision makers adopt a high / strict threshold. This appears to have two effects: first, the overall distribution is more tightly distributed near zero, indicating a lower degree of polarization among decision makers. This should not be too surprising: the mean of a larger number of values (pieces of information) will have less variability than the mean of a small number of values (pieces of information), meaning that participants will wind up closer to the true average distribution of information in the environment as they increase their thresholds. The second effect of thresholds is to moderate the prevalence of extremists. As illustrated in the figure, there are larger bumps (more extremists) when thresholds are low than when they are high. This occurs because the first and second pieces of information that participants gather are less likely to terminate the decision 
making process, because they are unlikely to push a decision maker far enough in one direction to pass a high threshold. As a result, the probability of stopping with very little information $(\operatorname{Pr}(t=1)=F(-\theta)+(1-F(\theta))$ in the proof above $)$ is much lower when $\theta$ is large. Thus, one potential way to reduce the prevalence of both polarization and extremism is to induce higher thresholds in decision makers, through interventions encouraging accuracy and thorough consideration of evidence and discouraging fast and inconsiderate choice strategies. However, the overall shape of the distribution of information in the population will never truly reflect the input distribution. There is always a gap between those favoring the option on the left and the option on the right, meaning that polarization is an unavoidable consequence of decision making when agents implement an optimal balance-of-evidence stopping rule.

Finally, the left panels of Figure 1 correspond to situations with no starting point bias, while the right panels correspond to situations with a starting point bias toward the right-hand (+) response. As shown, starting point bias has a quite substantial effect on the prevalence of extremists - whereas they constitute a relatively small proportion of decision makers even in the no bias conditions, the proportion of extremists nearly triples when start point bias goes from 0 (left) to 0.4 (right). This occurs because decision makers who already favor positive responses require very little information to reach the positive boundary - many decision makers will stop after receiving a single piece of information favoring the option that they are already biased toward. As a result, the prior distribution of beliefs among decision makers will have a drastic effect on extremism - a wide start point distribution will result in many extremists on both sides, while a narrow start point distribution will reduce the prevalence of extremists on both sides.

In one sense, this is quite intuitive: when people start with more extreme beliefs already, they make up their minds very quickly and are likely to to continue to hold extreme views. Our simulations additionally suggest that the decision making process will accentuate this extremism. Those that start with extreme views are likely to stop immediately after gathering information that makes them more extreme, and less likely to stop after sampling information that makes them less extreme. The decision making process therefore takes starting point biases and increases the bias 
in the population: the more decisions we make on related topics (where starting points could be expected to carry from one decision to the next), the more extreme our views become.

In many ways, this resembles interrogation and indoctrination strategies: have the subject admit to (decide in favor of) a small positive view, and then build on this small positive bias to make their views more extreme by iteratively offering them the chance to express (decide in favor of) more and more extreme views (Hinkle \& Wolff, 1956). The similarities between indoctrination and polarization by sequential decisions are chilling, and our simulations suggest that they probably draw on a common mechanism: starting point bias. Further work on polarization and extremism should therefore consider the interaction between prior biases and decision making a central mechanism, worthy of deep consideration and exploration.

\section{Multi-alternative choice}

One potential concern with the results we present here and in the main text is that we apply our analyses exclusively to binary choice. A critic, or even a well-meaning researcher hoping to determine avenues for interventions that reduce polarization, could look to multi-alternative choice as an unexplored possibility. We ourselves were hopeful that multi-alternative choice would curb polarization and extremism, thinking that this might provide support for eliminating two-party systems or for ranked-choice voting protocols as interventions.

Unfortunately, this is not the case - a similar pattern of polarization and extremism occurs beyond binary choice as well. Optimal choice strategies for these situations are provided in work by Link \& Heath (1975), Kvam (2019), and Tajima et al. (2019): essentially, a decision maker's beliefs in optimal trinary choice can be described by a location on a triangle, and by a multidimensional simplex when there are 4+ options (Kvam, 2019). To make a decision, the decision maker starts on the interior of the triangle (simplex) and gathers information, changing their beliefs accordingly until they cross one of the edges of the triangle (sides of a simplex). The size of the triangle (simplex) is determined by the height of the thresholds. This means that extreme information is still more likely that moderate information to terminate the decision 
process, with the end result being the same as in binary choice.

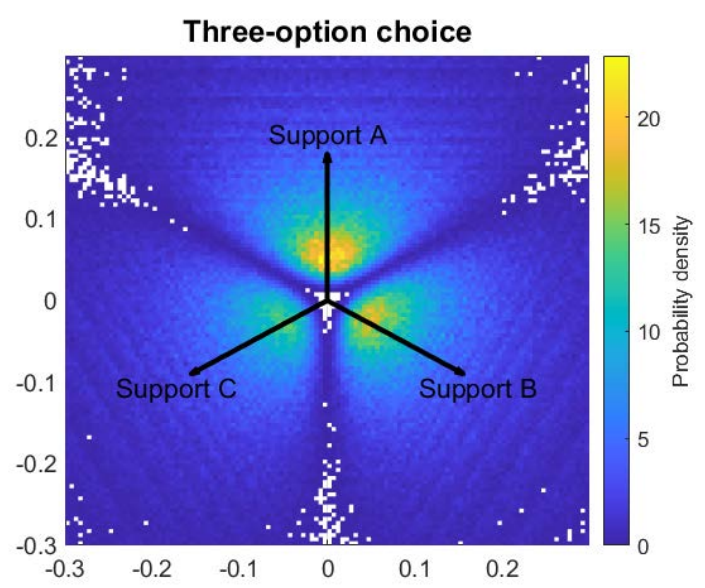

Figure 2. Distribution of beliefs (evidence) among a population of decision makers who have made a decision among three options $\mathrm{A}, \mathrm{B}$, and $\mathrm{C}$.

This is illustrated in Figure 2: a fully symmetric distribution of input choice evidence creates three "pockets" of posterior evidence favoring each option, centered around those favoring $\mathrm{A}$, those favoring B, and those favoring $\mathrm{C}$. There are very few decision makers between A and B, $\mathrm{B}$ and $\mathrm{C}$, and $\mathrm{A}$ and $\mathrm{C}$, and yet fewer who have a near-even view of all three. In fact, across more than 100,000 simulations shown in Figure 2, we had no instances of decision makers who had a balanced or even nearly-balanced view of the three options (white area of the figure). 


\section{References}

Bhatia, S., \& Mullett, T. L. (2018). Similarity and decision time in preferential choice. Quarterly Journal of Experimental Psychology, 71(6), 1276-1280.

Bogacz, R., Brown, E., Moehlis, J., Holmes, P., \& Cohen, J. D. (2006). The physics of optimal decision making: A formal analysis of models of performance in two-alternative forced-choice tasks. Psychological Review, 113(4), 700-765. doi: 10.1037/0033-295X.113.4.700

Diederich, A., \& Busemeyer, J. R. (2003). Simple matrix methods for analyzing diffusion models of choice probability, choice response time, and simple response time. Journal of Mathematical Psychology, 47(3), 304-322. doi: 10.1016/S0022-2496(03)00003-8

Diederich, A., \& Busemeyer, J. R. (2006). Modeling the effects of payoff on response bias in a perceptual discrimination task: Bound-change, drift-rate-change, or two-stage-processing hypothesis. Perception \& Psychophysics, 68(2), 194-207. doi: 10.3758/BF03193669

Edmunds, C. E., Bose, D., Camerer, C. F., Mullett, T. L., \& Stewart, N. (2020a). Accumulation is late and brief in preferential choice. PsyArXiv. doi: 10.31234/osf.io/sa4zr

Edmunds, C. E., Bose, D., Camerer, C. F., Mullett, T. L., \& Stewart, N. (2020b). Experimental investigations of attention interaction in preferential choice. https://osf.io/mvk95/.

Edwards, W. (1965). Optimal strategies for seeking information: Models for statistics, choice reaction times, and human information processing. Journal of Mathematical Psychology, 2(2), 312-329. doi: 10.1016/0022-2496(65)90007-6

Heitz, R. P. (2014). The speed-accuracy tradeoff: history, physiology, methodology, and behavior. Frontiers in Neuroscience, $8,150$.

Hinkle, L. E., \& Wolff, H. G. (1956). Communist interrogation and indoctrination of enemies of the states: Analysis of methods used by the communist state police (a special report). AMA Archives of Neurology \& Psychiatry, 76(2), 115-174. 
Kellen, D., Pachur, T., \& Hertwig, R. (2016). How (in) variant are subjective representations of described and experienced risk and rewards? Cognition, 157, 126-138.

Kvam, P. D. (2019). A geometric framework for modeling dynamic decisions among arbitrarily many alternatives. Journal of Mathematical Psychology, 91, 14-37.

Kvam, P. D., \& Pleskac, T. J. (2016). Strength and weight: The determinants of choice and confidence. Cognition, 152, 170-380. doi: 10.1016/j.cognition.2016.04.008

Link, S. W., \& Heath, R. A. (1975). A sequential theory of psychological discrimination. Psychometrika, 40(1), 77-105. doi: 10.1007/BF02291481

Mulder, M. J., Wagenmakers, E.-J., Ratcliff, R., Boekel, W., \& Forstmann, B. U. (2012). Bias in the brain: a diffusion model analysis of prior probability and potential payoff. Journal of Neuroscience, 32(7), 2335-2343.

Mullett, T. L., Ravichandar, N., \& Stewart, N. (in prep). Attention and accumulation in risky choice. https://osf.io/mvk95/.

Mullett, T. L., \& Stewart, N. (2016). Implications of visual attention phenomena for models of preferential choice. Decision.

Pleskac, T. J., Yu, S., Hopwood, C., \& Liu, T. (2019). Mechanisms of deliberation during preferential choice: Perspectives from computational modeling and individual differences. Decision, 6(1), 77-107.

Plummer, M. (2003). JAGS: A program for analysis of Bayesian graphical models using Gibbs sampling. In Proceedings of the 3rd international workshop on distributed statistical computing (Vol. 124, p. 10).

Ratcliff, R. (1978). A theory of memory retrieval. Psychological Review, 85(2), 59-108. doi: 10.1037/0033-295X.85.2.59 
Ratcliff, R., Smith, P. L., Brown, S. D., \& McKoon, G. (2016). Diffusion decision model: Current issues and history. Trends in cognitive sciences, 20(4), 260-281.

Shimojo, S., Simion, C., Shimojo, E., \& Scheier, C. (2003). Gaze bias both reflects and influences preference. Nature neuroscience, 6(12), 1317-1322.

Tajima, S., Drugowitsch, J., Patel, N., \& Pouget, A. (2019). Optimal policy for multi-alternative decisions. Nature neuroscience, 22(9), 1503-1511.

Turner, B. M., Gao, J., Koenig, S., Palfy, D., \& McClelland, J. L. (2017). The dynamics of multimodal integration: The averaging diffusion model. Psychonomic bulletin \& review, 24(6), 1819-1843.

Wickelgren, W. A. (1977). Speed-accuracy tradeoff and information processing dynamics. Acta Psychologica, 41(1), 67-85. doi: 10.1016/0001-6918(77)90012-9 